\title{
STOCHASTIC GENERATION OF DAILY GROUNDWATER LEVELS BY ARTIFICIAL NEURAL NETWORKS
}

\author{
Camilo A. S. de FARIAS ${ }^{1}$, Akihiro KADOTA², Koichi SUZUKI ${ }^{3}$ and \\ Kuzue SHIGUEMATSU4 \\ ${ }^{1}$ Dr. of Eng., Associate Professor, Academic Unit of Env. Science and Technology, Federal Univ. of Campina Grande \\ (Rua Cel João Leite 517, Pombal, Paraíba 58840-000, Brazil) \\ E-mail: camiloallyson@yahoo.com.br \\ ${ }^{2}$ Member of JSCE, Dr. of Eng., Associate Professor, Dept. of Civil and Environmental Engineering, Ehime University \\ (3 Bunkyo-cho, Matsuyama, Ehime 790-8577, Japan) \\ 3 Fellow of JSCE, Dr. of Eng., President, Niihama National College of Technology \\ (7-1 Yagumo-cho, Niihama, Ehime 792-8580, Japan) \\ 4 Member of JSCE, Technical Official, Dept. of Civil and Environmental Engineering, Ehime University \\ (3 Bunkyo-cho, Matsuyama, Ehime 790-8577, Japan)
}

\begin{abstract}
Many studies have been carried out in order to produce measures capable of ensuring the sustainable use of the water resources. A better management of water resources systems can be achieved if the long term information of hydrologic variables is available, which is not always the case. Stochastic simulation of such hydrologic variables is an attractive alternative to extend the length of observed records. This paper applies an Artificial Neural Network (ANN) model for simulating daily groundwater levels for the city of Matsuyama, Japan. The stochastic generated series must keep not only the statistical properties but also the seasonal oscillations of the observed series. Ten years of observed daily data were used for calibrating the model parameters. The results show that the model preserves the major statistical properties.
\end{abstract}

Key Words: Stochastic simulation, artificial neural networks, groundwater level.

\section{INTRODUCTION}

The insufficiency of water has become a periodical difficulty for many cities around the world, mainly due to reduced water availability and increasing population. As a result, several researches have been carried out in order to produce measures capable of ensuring the sustainable use of the water resources. Matsuyama City, located in southwestern part of Japan, is one of these cities and many optimization techniques have been studied in a tentative of improving and finding adequate policies for its water resource system, which is composed of a dam reservoir and a set of unconfined groundwater wells $^{1), 2)}$. However, for the implementation of such techniques, it is frequently necessary to use long scenarios of daily reservoir inflows and groundwater levels.

Stochastic simulation of hydrologic variables is an attractive alternative to extend the length of observed records ${ }^{3), 4), 5), 6), 7)}$. The generation of scenarios is generally carried out by models that try to produce synthetic data having statistical properties as close as possible to the observed series.

Implicit stochastic optimization (ISO) procedures are going to be used for the optimal operation of Matsuyama City's groundwater system. The ISO procedure implicitly considers the variability of the system's variables by using long series of historical or synthetic values into a deterministic optimization model ${ }^{8), 9)}$. This work investigates the use of an Artificial Neural Network (ANN) model for the stochastic simulation of daily groundwater levels in the city of Matsuyama. The parameters of the model are calibrated and used for generating stochastic scenarios, which have their statistical properties compared to the observed ones.

The network employed in this study is an input 
delayed artificial neural network (IDNN). IDNNs are dynamic artificial neural networks capable of accounting for nonlinearities and representing temporal information of input sequences, which make them very suitable for time series modeling ${ }^{10}$.

\section{STUDY SYSTEM}

Matsuyama City water system is composed of a multipurpose reservoir and a set of unconfined wells located around Shigenobu River, which is the main river of its hydrographic basin. The groundwater of Shigenobu River together with Ishitegawa Dam reservoir is used for supplying all the water needs of the city. Ishitegawa Dam is also used for irrigation and flood control in the region.

Shigenobu River Basin has an area of approximately $445 \mathrm{~km}^{2}$, an annual precipitation of around $1,250 \mathrm{~mm}$ and about half a million residents. The layout and location of the water supply system of Matsuyama city is shown in Fig. 1.

The set of unconfined wells located are responsible

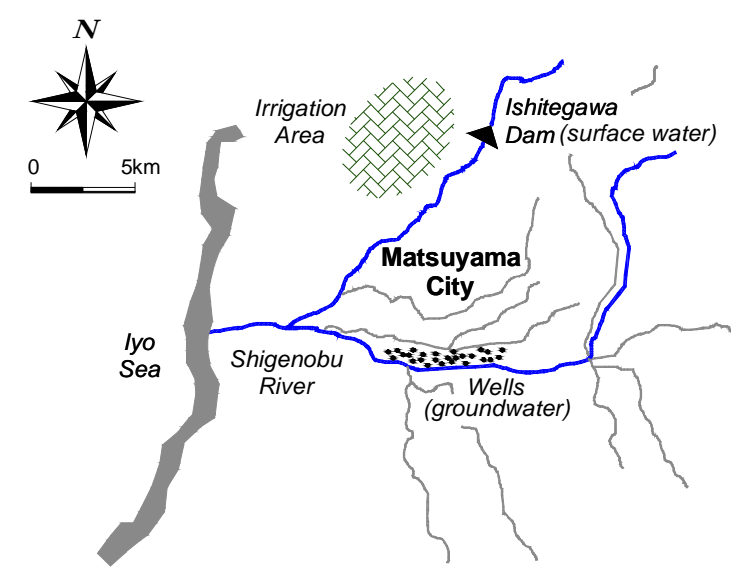

Fig. 1 Location and layout of the system.

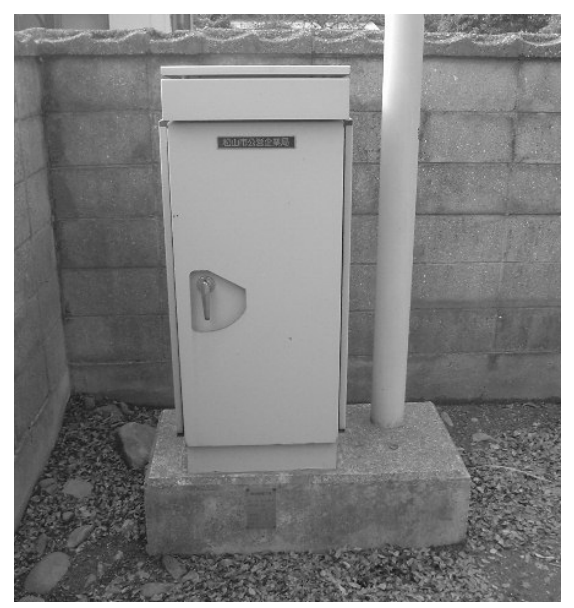

Fig. 2 Minamitakai observation well. for supplying part of Matsuyama City's domestic demand. The operation of these wells is based on the water table measures carried out at the Minamitakai observation well, which is shown in Fig. 2.

\section{INPUT DELAYED NEURAL NETWORK (IDNN) MODEL}

IDNNs contain two components: a memory and an associator. The memory is responsible for holding the past information, which in this study is composed of the two previous groundwater levels. The associator is a multilayer perceptron network that relates the memory with the desired output, i.e., the current groundwater level. Thus, the memory component represents the temporal information and the associator accounts for the nonlinearity, making the IDNNs very suitable for time series modeling.

\section{(1) Architecture}

The architecture of the network is formed by the input layer, one hidden layer and the output layer. The input layer is composed of two neurons, which are the two previous groundwater levels. The number of neurons in the hidden layer is determined based on a trial-error procedure. The best training results were achieved with three neurons in the hidden layer. The current groundwater level is the single neuron of the output layer.

\section{(2) Topology}

For neural networks, not only the way neurons are implemented but also how their interconnections (topology) are made is important. In this study the network topology is constrained to be feed-forward, i.e., the connections are allowed from the input layer to the hidden layer and from the hidden layer to the output layer. Fig. 3 illustrates the network topology of this study.

In this network, each element of the input vector is connected to each neuron in the hidden layer. The $i$ th neuron in the hidden layer has a summation that gathers its weighted inputs and bias to form its own scalar output or induced local field. Each induced local field is submitted to an activation function so that they become the inputs of the output layer. The unique neuron in the output layer also has a summation that gathers its weighted inputs (from the hidden layer) and bias to form its induced local field. This induced local field is then submitted to the neuron activation function and becomes the final output.

\section{(3) Activation functions}

Continuous and differential functions are necessary 
for relating inputs and outputs of the artificial neural networks. According to Haykin ${ }^{11)}$, the sigmoid function, which is a mathematical function having an " $S$ " shape, is a good activation function for each neuron due to its generally accepted behavior. The tan-sigmoid function shown in Eq. 1 is chosen as the activation function for the hidden neurons. For the output layer neuron, a linear activation function is used.

$$
\phi(a)=\frac{2}{\left(1+e^{-2 a}\right)}-1
$$

where $a$ is the input to the tan-sigmoid activation function $\phi$.

\section{(4) Training process}

The original data (input and desired outputs) are conveniently scaled before the training in order to improve the efficiency of the IDNN ${ }^{12}$ ). The scaling function scales the inputs and targets of the IDNN so that they fall in the range $[-1,1]$ as shown in Eq. 2.

$$
k^{\prime}(t)=2\left(\frac{k(t)-k_{\min }}{k_{\max }-k_{\min }}\right)-1
$$

where $t$ is the time index; $k$ is the input or target to be scaled; $k_{\max }$ and $k_{\min }$ are the maximum and minimum values in the series of known data used for calibrating the IDNN, respectively; and $k$ ' is the scaled value of the input or output $k$.

The training is performed by a back-propagation algorithm which has been successfully applied to water resources systems. In this approach, the Scaled Conjugate Gradient (SCG) method is used for the back-propagation. A detailed explanation of the SCG method is provided by Moller ${ }^{13)}$. The network training is supervised, i.e., the series of weights between the neurons and the bias are adjusted through the iterations (epochs) in order to fit the series of inputs to another series of known outputs. The training also occurs in the batch mode. In this mode the weights and biases are updated only after the entire training set has been applied to the network.

In order to improve generalization, the training is stopped by the Early Stopping Method ${ }^{12)}$. The Early Stopping Method divides the calibration data into two subsets: training and validation. The training subset is used for calculating gradients and adjusting weights and biases. The validation subset, on the other hand, has its errors monitored in order to avoid overfitting. The training is stopped when the error on the validation set increases for a certain number of iterations.

\section{(5) Generation of the synthetic series}

The synthetic daily groundwater levels are obtained by two components: one is deterministic and the other is stochastic.

\section{a) Deterministic component}

The IDNN results are the deterministic component for the generation of synthetic groundwater levels. The IDNN series of outputs are obtained from the calibrated IDNN model operation as follows:

$$
\begin{gathered}
a_{i}(t)=\left[\sum_{j=1}^{I N=2} x_{j}(t) \times w_{i, j}\right]+b_{i} \\
o^{\prime}(t)=\phi_{2}\left\{\left\{\sum_{i=1}^{H N=3} \phi_{1}\left[a_{i}(t)\right] \times w_{h, i}\right\}+b_{h}\right\} \\
O^{\prime}=\left\{o^{\prime}(1), o^{\prime}(2), \cdots, o^{\prime}(n)\right\}
\end{gathered}
$$

where $i$ is the neuron index in the hidden layer; $a_{i}(t)$ is the induced local field of the ith neuron in the hidden layer at time $t ; x_{j}(t)$ is the value of the $j$ th neuron in the input layer at time $t$; $I N$ is the number of input neurons; $w_{i, j}$ is the weight between the $j$ th input neuron and the destination neuron $i ; b_{i}$ is the bias of the $i$ th neuron in the hidden layer; $o$ ' $(t)$ is the IDNN output at time $t ; \phi_{2}$ is the linear activation function for the neuron in the output layer; $H N$ is the number of hidden neurons; $\phi_{1}$ is the sigmoid activation function for all neurons in the hidden layer; $w_{h, i}$ is the weight between the ith input neuron (from hidden layer) and the destination neuron $h$; $b_{h}$ is the bias of the output layer neuron; $O$ ' is the set of results from the IDNN model; and $n$ is the number of days to be generated by the model. The deterministic component is then obtained by applying the inverse of the scaling function:

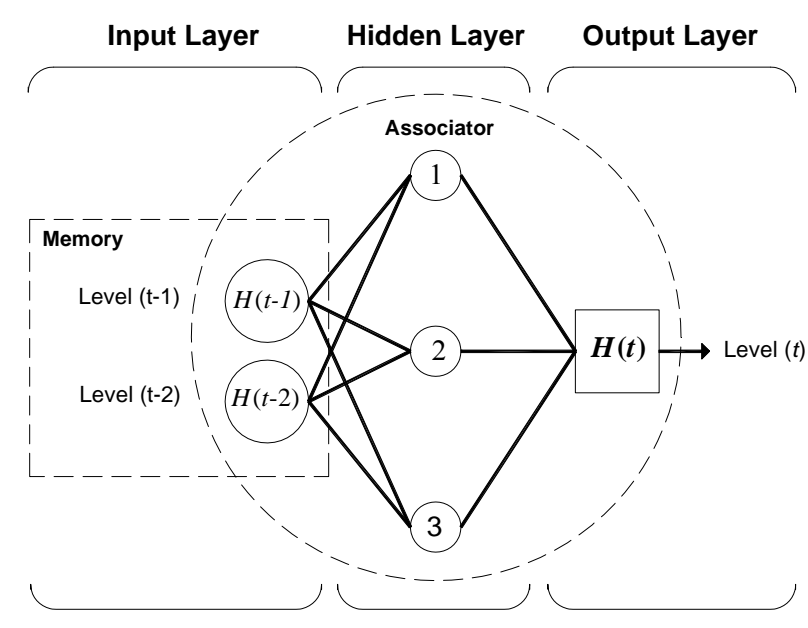

Fig. 3Topology of the IDNN. 


$$
\begin{gathered}
o(t)=\left(\frac{o^{\prime}(t)+1}{2}\right) \times\left(k_{\max }-k_{\min }\right)+k_{\min } \\
O=\{o(1), o(2), \cdots, o(n)\}
\end{gathered}
$$

in which $o(t)$ is the output for the inverse of the scaling function at time $t$; and $O$ is the deterministic component.

\section{b) Stochastic component}

When the set of standardized IDNN outputs and observed results are compared, the difference between them leads to a residuals series. Statistical analysis of the residuals shows that they can be modeled as normal distributions. A fitting of the residuals series is provided, and the posterior random generation of these values is the stochastic component of the model.

\section{c) Groundwater level series generation}

The synthetic groundwater level series is found by the following equations:

$$
\begin{gathered}
r=\{r(1), r(2), \cdots, r(n)\} \\
H_{S}(t)=o(t)+r(t) \\
S H_{S}=\{H(1), H(2), \cdots, H(n)\}
\end{gathered}
$$

where $r$ is the series of random generated residuals obtained from a properly fitted normal distribution; $r(t)$ is the residual at time $t ; H_{S}(t)$ is the synthetic groundwater level at time $t$; and $S H_{S}$ is the series of synthetic daily groundwater levels.

Like the fitting and generation of residuals series, the IDNN model was implemented in MATLAB environment.

\section{GENERATION OF STOCHASTIC SERIES}

The IDNN model was applied for generating synthetic daily groundwater levels in the Minamitakai well. This well is used as basis to operate a set of unconfined well that supplies water to the city of Matsuyama, located in Ehime, Japan. For practical purposes, the maximum release capacity from the set of groundwater wells is estimated as shown in Fig. 4. Ten years (1991-2000) of observed daily groundwater levels were used for calibrating (1991-1995 for training and 1996-2000 for validation) the IDNN model. After the determination of the deterministic component by Eqs. (3-7), the synthetic series were obtained by the random generation of the fitted normal distribution applied to Eqs. (8-10).

In order to check the performance of the IDNN model, 5 synthetic series of daily groundwater levels (each 10-year long) were generated and its statistics were compared with those from the observed data.

Measures of location (mean) and spread (standard deviation) are often used to classify a new set of data. These two types of measures represent the first statistical moments of a dataset. Location represents the general magnitude of the data values and spread refers to the degree of dispersion around the central value $^{14)}$.

Major statistical properties such as daily mean and standard deviation were compared with those from the observed series. Fig. $\mathbf{5}$ shows the errors between simulated and observed daily means and standard deviations for groundwater levels.

Comparison of monthly means and standard deviations of the observed series with the ones found by the IDNN model are shown in Fig. 6. The histograms of observed and generated series are presented in Fig. 7. Annual statistics for observed and synthetic series are presented in Table 1.

The results showed that the daily and monthly statistics found by the IDNN model were very close to those from the observed data set. The histograms of daily groundwater levels were also shown to be very similar to their observed counterparts.

Analysis of all results reveals that the IDNN model could preserve not only the statistical properties but also the seasonal oscillations of the observed data.

\section{SIMULATION OF SCENARIOS}

Optimization techniques for the operation of Matsuyama groundwater system are going to be

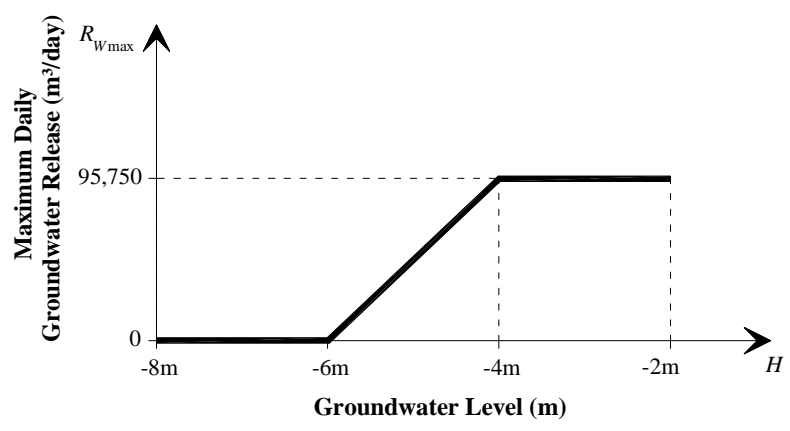

Fig. 4 Maximum daily groundwater release as a function of groundwater level at the observation well.

Table 1 Annual statistics for historical and synthetic series.

\begin{tabular}{|lcc|}
\hline \multicolumn{1}{|c|}{ Series } & Mean (m) & Standard Deviation (m) \\
& & \\
\hline Observed & $-2,67$ & 0,66 \\
IDNN & $-2,68$ & 0,69 \\
\hline
\end{tabular}




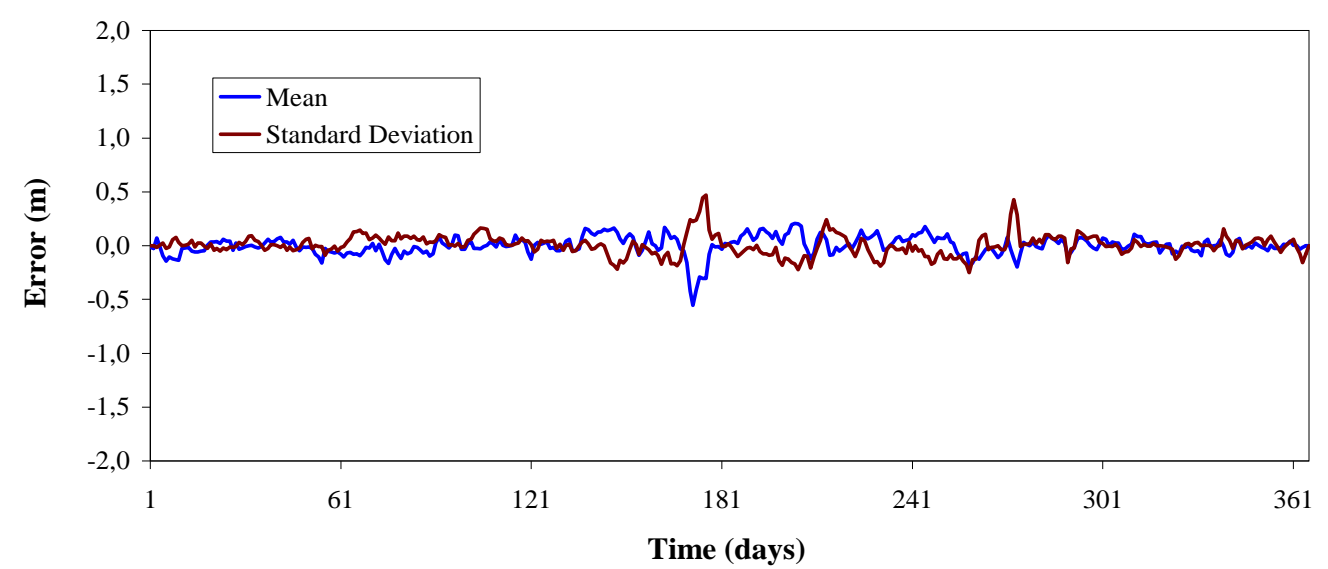

Fig. 5 Errors between simulated and observed daily means and standard deviations for groundwater levels.
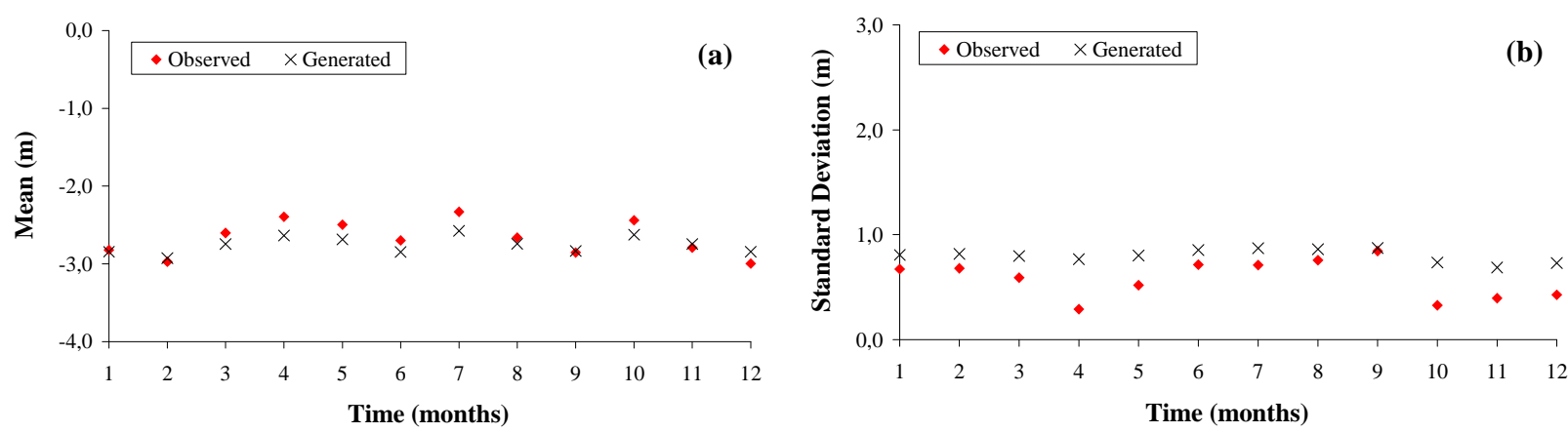

Fig. 6 Comparison of monthly (a) mean and (b) standard deviation of the observed series with the ones found by the IDNN model.
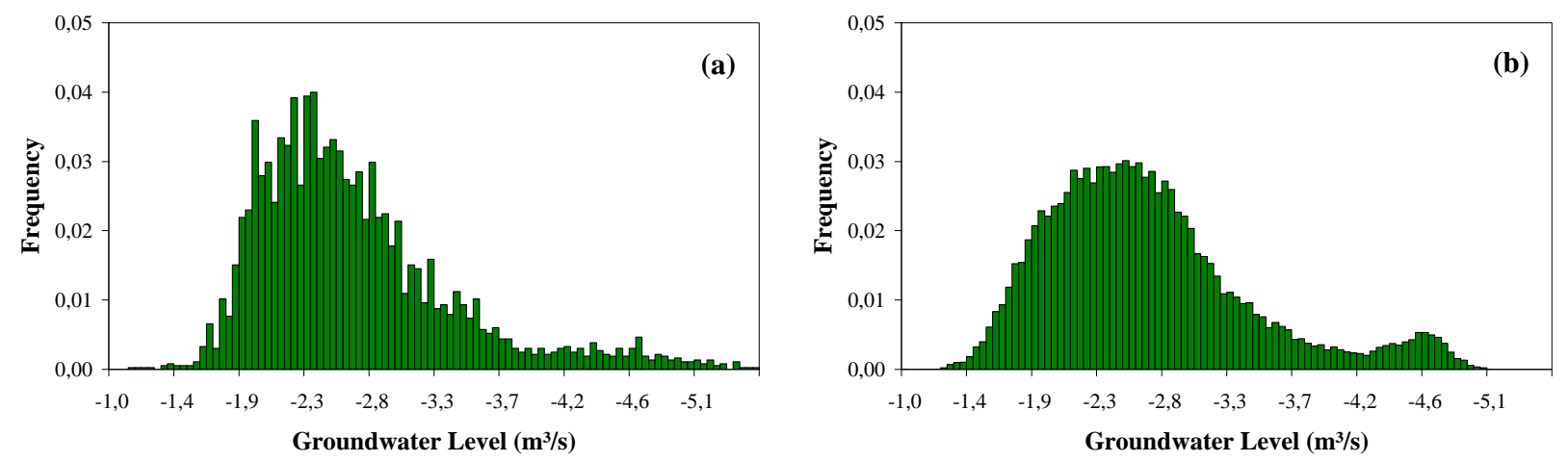

Fig. 7 Histograms of groundwater levels for (a) observed and (b) generated series.

tested with possible groundwater level scenarios.

Stochastic groundwater level scenarios were generated by the IDNN model to show the different possibilities of groundwater levels occurrence. As an example, Figs 8-9 display data for 2-scenario generations of 10-year length each. The observed groundwater levels are provided for comparison.

Any number of possible stochastic scenarios can be provided by the IDNN model. The statistical properties results indicate that this model is very useful for producing trustworthy inputs to the optimization techniques used in Matsuyama's water resources system.

\section{CONCLUSIONS}

A model based on Artificial Neural Networks was developed and applied for generating stochastic groundwater levels for the city of Matsuyama, Japan. These scenarios are going to be used as inputs to optimization procedures in a tentative of improving and finding more adequate policies to the water resources system of this city.

The statistics (means and standard deviations) obtained by the model were very close to the ones presented in the observed data set. The histograms of simulated groundwater levels were also very similar to those from the observed data set.

As conclusion, the results suggest that the IDNN 


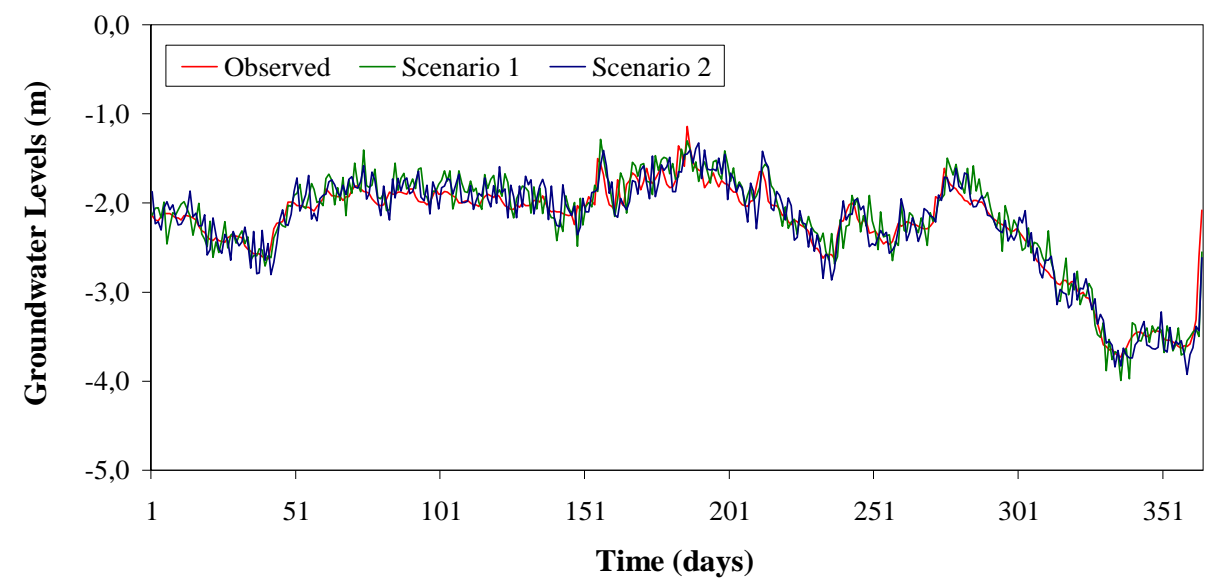

Fig. 8 Sample of generated scenarios for 1991 by the IDNN model.

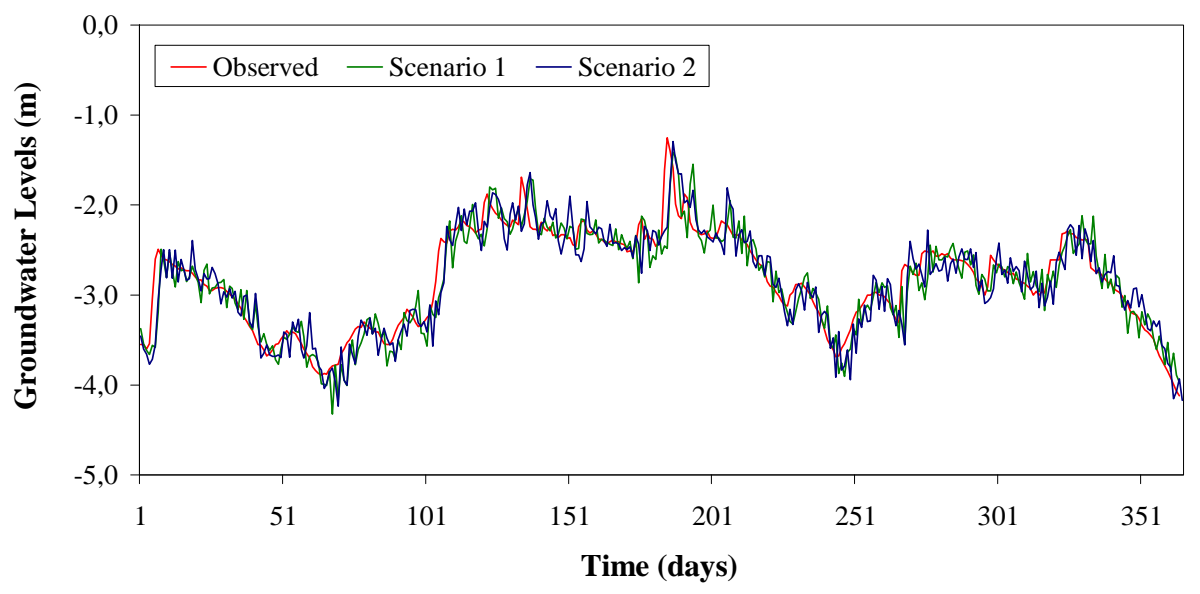

Fig. 9 Sample of generated scenarios for 1995 by the IDNN model.

model is suitable for simulating the daily groundwater level scenarios needed by the implicit stochastic optimization technique for the optimal operation of Matsuyama City's groundwater system.

\section{REFERENCES}

1) Celeste, A. B., Suzuki, K., Kadota, A., Farias, C. A. S.: Stochastic generation of inflow scenarios to be used by optimal reservoir operation models, Annual Journal of Hydraulic Engineering, JSCE, Vol. 48, pp. 451-456, 2004.

2) Farias, C. A. S., Celeste, A. B., Sakata, Y., Kadota, A., Suzuki, K.: Use of monte carlo optimization and artificial neural networks for deriving reservoir operating rules, Annual Journal of Hydraulic Engineering, JSCE, Vol. 50, pp. 25-30, 2006.

3) Yevjevich, V. Stochastic processes in hydrology. Water Resources Publication, Fort Collins, Colorado, 1972.

4) Reddy, P. J. Stochastic hydrology. Laxmi Publications, Delhi, 1987.

5) Salas, J. D.: Analysis and modeling of hydrologic time series, In: Maidment, D. R.: Handbook of Hydrology, McGraw-Hill, Inc., New York, pp. 19.1-19.72, 1993.

6) Thomas, H. A., \& Fiering, M. B. Mathematical synthesis of stream-flow sequences for the analysis of river basins by simulation. in: A. Maass et al. (ed) Design of Water Resource
Systems. Harvard Univ. Press, Cambridge, Massachusetts, pp. 59-493, 1962.

7) Richardson, C. W.: Stochastic simulation of daily precipitation, temperature, and solar radiation, Water Resources Research, 17(4), pp. 182-190, 1981.

8) Nandalal, K. D. W. \& J. J. Bogardi: Dynamic Programming Based Operation of Reservoirs: Applicabitity and Limits, Cambridge University Press, United Kingdom, 2007.

9) Young, G. K., Jr.: Finding reservoir operating rules, Journal of the Hydraulic Division, ASCE, 93(HY6), pp. 297-321, 1967.

10) Coulibaly, P., Anctil, F., Bobée, B.: Multivariate reservoir inflow forecasting using temporal neural networks, Journal of Hydrologic Engineering, ASCE, Vol. 6, pp. 367-376, 2001.

11) Haykin, S.: Neural networks: a comprehensive foundation, Prentice Hall, Inc., 2.ed, New Jersey, 1999.

12) Demuth, H. \& Beale, M.: Neural Network Toolbox: for use with Matlab. The MathWorks, Inc., Natick, USA, 2005.

13) Moller, M. F.: Scaled conjugate gradient algorithm for fast supervised learning, Neural Networks, Vol. 6 (4), pp. 525-533, 1993.

14) Wilks, D. S. Statistical methods in the atmospheric sciences, Elsevier Academic Press Publications, 2. ed., San Diego, 1995.

(Received September 30, 2010) 